\title{
Scarce for Me and Popular for Others: The Impact of Scarcity Versus Popularity Cues on Self Versus Other Decision-Making (An Abstract)
}

\section{Christopher Lee and Laurie Wu}

\begin{abstract}
Scarcity and popularity cues are two pervasively used promotional strategies in today's marketplace. While some ads include scarcity information such as "limited edition," others denote products as popular with information cues such as " $75 \%$ of consumers viewed this page purchased our product." Yet, little research has been devoted to compare the effectiveness of these two cue types (Deval et al. 2013; Steinhart and Kamins 2014). As marketers, we do not fully understand in what situation to use a scarcity cue vs. a popularity cue in promotional campaigns. The current research adds to this growing stream of knowledge by proposing recipient (i.e., buying for self vs. other) as an important contextual variable that determines the relative effectiveness of scarcity vs. popularity cues. Findings of our research will provide important insights for marketing researchers and practitioners in both online and offline marketing.
\end{abstract}

References Available Upon Request

C. Lee $(\bowtie) \cdot$ L. Wu

Temple University, Philadelphia, PA, USA

e-mail: chrislee@temple.edu; laurie.wu@temple.edu 